\title{
Application of two-dimensional sandwich structure supported Pt single-atom catalyst in photocatalytic hydrogen evolution: first principle
}

yanshuai li ${ }^{1}$, shizhi dong ${ }^{1}$, bingshuai $\mathrm{zhu}^{1}$, ruichuan $\mathrm{li}^{1}$, wenlong shang ${ }^{1}$, linghui chen $^{1}$, jinyu zhang ${ }^{1}$, zhilong zhao ${ }^{1}$, and lin guo ${ }^{2}$

${ }^{1}$ Liaoning Technical University

${ }^{2}$ Beihang University

May 26, 2021

\begin{abstract}
The preparation of hydrogen by photocatalytic principle is an important subject in the future energy direction. This article intends to design the catalytic structure of a crystalline (amorphous) TiO2/two-dimensional MoS2 sandwich structure supporting a single-atom, and explore the effect of this special structure on the photocatalytic reaction.Calculating the effective mass, electrostatic potential, light absorption spectrum and other related properties by DFT method, the analysis shows that the sandwich structure catalyst has good carrier transfer ability and electron-hole separation ability. Except for the $\mathrm{Pt} @$ Amorphousr-TiO2/MoS2 sandwich structure single-atom catalyst, which only has an excellent effect on the oxygen evolution of water, the rest of the interlayer catalysts have the two characteristics of photocatalytic hydrogen evolution and oxygen evolution. The new catalyst designed in this paper has innovative design ideas and high-efficiency research and development value.
\end{abstract}

\section{Hosted file}

Manuscripts.pdf available at https://authorea.com/users/415888/articles/523650application-of-two-dimensional-sandwich-structure-supported-pt-single-atom-catalystin-photocatalytic-hydrogen-evolution-first-principle 